\title{
Risk Factors for Fungemia in Very Low Birth Weight Infants
}

\section{Maria Gibelli and Vera Krebs*}

Department of Pediatrics, School of Medicine, Sao Paulo University, Brazil

\begin{abstract}
Fungemia is a severe complication on neonatal period among very low birth weight infants (VLBW). The aim of the study was to describe the incidence of fungal sepsis and to analyze the risk factors among this population. Data were collected prospectively over a 25 months period among VLBW infants admitted at the Neonatal Intensive Care Unit (NICU), with more then 72 hours of life. Patients were divided in five groups, based on the first positive blood culture: without sepsis; sepsis with negative blood culture; Gram-positive bacterial sepsis; Gram-negative bacterial sepsis and fungal sepsis. For statistical analyses the Pearson test, the Kruskal-Wallis test and a logistic regression model were used. 187 newborns were included in the study: $110(58,8 \%)$ had late-onset sepsis; $13(7 \%)$ had fungal sepsis. Rate of mortality was $69,2 \%$. The risk factors identified at the comparison between the fungemia group and no-fungemia (all the remaining sample) were: birth weight (BW), gestational age (GA), central venous catheter (CVC), parenteral nutrition (PN), fasting, mechanical ventilation (MV), exposure to vancomycine, cefepime, meropenem, and amikacine. Further statistical analyses have shown: for each increasing of $10 \mathrm{~g}$ in BW, the risk of fungemia diminished $3 \%$; each day of CVC, increased this risk in $8,1 \%$; each day of $M V$ increased this risk in $11,1 \%$. The analyses of the categorized variables have shown: BW $1000 \mathrm{~g}$ increased the risk of fungemia 23 times; MV 14 days increased the risk 36 times; each day of CVC increased the risk of fungemia in 9,3\%.
\end{abstract}

Keywords: Risk factors; Fungemia; Very low birth weight infants

\section{Introduction}

Candida $s p p$ is an important agent of late-onset sepsis in very low birth weight infants (VLBW) associated with elevated morbidity and mortality [1-8]. Clinical manifestations are nonspecific, and the suspicion of Candida infection among VLBW infants can be very helpful for diagnosis.

In 1996, Stoll et al. noted that Candida spp was identified in $9 \%$ of the VLBW infants admitted in their study. Six years later, the same group described its prevalence as $12 \%$, still the third most important agent of LOS but presenting an increase in the number of cases [1-2]

Kossof et al. noted that $70 \%$ of newborns with fungemia had BW $<1000 \mathrm{~g}$ (median=765 g) and GA $<28$ weeks (median=26 weeks) [4]. Fridkin et al. showed that the incidence of Candida spp infections was higher between newborns with $\mathrm{BW}<1000 \mathrm{~g}$ [6]. Clerihew et al. observed that the BW median between newborns with fungemia was $720 \mathrm{~g}$ and $86 \%$ of the sample corresponded to extremely low birth weight infants, with a median GA $=25$ weeks [7]. The increasing number of VLBW infants admitted in the NICU with fungal sepsis shows that risk factors may play an important role in developing the disease. The most important risk factors in the NICU are mechanical ventilation $(\mathrm{MV})$, central venous catheters (CVC), broad-spectrum antibiotics, gestational age (GA) of less than 28 weeks, low birth weight, fasting, parenteral nutrition (PN) and lipids, use of antagonists of histamine, and steroids and abdominal surgeries [9-12].

The use of CVC in a VLBW infant is an important risk factor for fungal sepsis. The very composition of the catheter constitutes an aspect for developing Candida sepsis. The material used to produce these catheters allows the aggregation of the biofilm and protein matrix, easing the aggregation of bacteria and Candida [13-15]. The PN is a nutritional substract of microorganisms. The presence of lipids and the continuous infusion of glucose are some of the characteristics that facilitate fungal infection [16].

Do any of these particular risk factors play a special role in developing fungal sepsis? The aim of this study is to analyze each risk factor among VLBW infants admitted to a level III NICU.

\section{Methods}

We performed a prospective study at a level III NICU of a university teaching hospital to determine the risk factors for fungemia in VLBW infants admitted from June 1, 2005 to June 30, 2007. Hospital das Clinicas is a 2,000-bed tertiary-care university hospital affiliated with the University of São Paulo, Brazil. It has a neonatal unit with 63 beds that accepts only babies born in the hospital, usually from high-risk pregnancies. All NICU VLBW infants with age $>72$ hours were enrolled and infants staying for $\leq 72$ hours were excluded from the study.

The following variables were analyzed: gender, BW (g), GA (weeks), fasting (days), MV (days), CVC (days), PN (days), broadspectrum antibiotics (days), and outcome (death or discharge). We considered sepsis as the suspicion of infection and at least two of the following four criteria, one of which must be abnormal temperature or leukocyte count: fever/hypothermia, leucocytosis/leucopenia or $>10 \%$ of immature neutrophils, tachycardia/bradycardia, tachypnea/apnea [17] We considered the first episode of late-onset sepsis and the first positive blood culture obtained. Newborns were distributed according to the microorganism found: 1) no sepsis; 2) sepsis with negative blood culture; 3) gram-positive bacterial sepsis; 4) gram-negative bacterial sepsis; and 5) fungal sepsis. One patient was included in the fungal sepsis group after an autopsy finding.

\section{Statistical Analysis}

To analyze the possible association between the variables and

*Corresponding author: Vera Krebs, Department of Pediatrics, School of Medicine, Sao Paulo University, Brazil, Tel: 5511 30781975; E-mail: verakrebs@uol.com.br

Received June 17, 2013; Accepted July 12, 2013; Published July 15, 2013

Citation: Gibelli M, Krebs V (2013) Risk Factors for Fungemia in Very Low Birth Weight Infants. Clin Microbial 2: 122. doi:10.4172/2327-5073.1000122

Copyright: ( 2013 Gibelli M, et al. This is an open-access article distributed under the terms of the Creative Commons Attribution License, which permits unrestricted use, distribution, and reproduction in any medium, provided the original author and source are credited. 
the groups, we realized the Pearson test, the chi-squared test, and the Kruskal-Wallis test $(\mathrm{p} \leq 0.05)$. To make multiple comparisons between the groups, we used Bonferroni's Test $(\mathrm{p} \leq 0.005)$. A multinomial logistic regression model was adjusted to the data to measure the effect of the significant values present in fungal infection compared with the absence of fungal infection. Another multinomial logistic regression was used with the categorized variables: BW $(>1000 \mathrm{~g}$ and $\leq 1000 \mathrm{~g})$, GA ( $>28$ weeks and $\leq 28$ weeks), fasting ( $>7$ days and $\leq 7$ days), $\mathrm{PN}(>7$ days and $\leq 7$ days), and MV (> 14 days and $\leq 14$ days). The following software was used: MSOffice Excel version 2000 and SPSS for Windows version 12.0 - Statistical Package for the Social Science. The study was approved by the institutional Ethical Committee.

\section{Results}

One hundred and eighty seven VLBW infants were included; 110 (58.8\%) had late-onset-sepsis; 57 (30.7\%) had negative blood culture;
$24(12.8 \%)$ had gram-positive sepsis; 16 (8.5\%) had gram-negative sepsis; and $13(7 \%)$ had fungal sepsis. The mortality rate for fungal sepsis was $69.2 \%$. We identified C.albicans (6 cases, 50\%), C.parapsilosis ( 5 cases, $41.7 \%$ ); and no-identified Candida (1 case, 8.3\%). One patient (8.3\%) was included based on autopsy findings. The characteristics of each group are shown in Table 1.

The comparison of the risk factors considering the presence and the absence of fungal infection and the comparison between the groups (Table 2), showed that all variables have a significant association with fungal sepsis. BW, GA, fasting, NPP, VPM, CVC, vancomycin, meropenem or cefepime were significantly associated with fungal sepsis in comparison with the group without sepsis. Likewise, fasting, NPP and CVC were significantly associated with fungal sepsis in comparison with the group with sepsis and negative blood culture.

To evaluate the effects of the variables present in fungal infection, we developed an initial model of multinomial logistic regression

\begin{tabular}{|c|c|c|c|c|c|}
\hline \multirow[b]{2}{*}{ Variable } & \multicolumn{5}{|c|}{ Group } \\
\hline & No sepsis & Sepsis and negative blood culture & G+ sepsis & G- sepsis & Fungal sepsis \\
\hline$B W(g)$ & $\mathrm{n}=77$ & $n=57$ & $n=24$ & $n=16$ & $n=13$ \\
\hline Mean & 1221.95 & 926.4 & 1102.5 & 936.25 & 869.23 \\
\hline Median & 1260 & 870 & 1105 & 955 & 910 \\
\hline Min -max & $580-1490$ & $530-1470$ & $730-1490$ & $530-1400$ & $410-1340$ \\
\hline \multicolumn{6}{|c|}{ GA (weeks) } \\
\hline Mean & 31.752 & 29.335 & 30.338 & 30.894 & 28.117 \\
\hline Median & 32 & 28.5 & 30.1 & 30 & 28.4 \\
\hline Min -max & $24.2-37$ & $24.3-36.1$ & $25.9-36.8$ & $25.9-38$ & $25.9-29.9$ \\
\hline \multicolumn{6}{|c|}{ Fasting (days) } \\
\hline Mean & 3.59 & 10.25 & 12.67 & 7.25 & 19.15 \\
\hline Median & 3 & 7 & 9 & 6,5 & 18 \\
\hline Min-max & $0-27$ & $0-50$ & $1-74$ & $1-19$ & $6-51$ \\
\hline \multicolumn{6}{|l|}{ NP (days) } \\
\hline Mean & 7.4 & 20.6 & 21.96 & 19.5 & 31.15 \\
\hline Median & 6 & 16 & 20 & 15.5 & 32 \\
\hline Min-max & $0-63$ & $0-70$ & $1-80$ & $1-54$ & $12-48$ \\
\hline \multicolumn{6}{|l|}{ MV (days) } \\
\hline Mean & 2.1 & 20.81 & 20.38 & 15.13 & 33 \\
\hline Median & 0 & 11 & 11.5 & 15.5 & 37 \\
\hline Min-max & $0-26$ & $0-150$ & $0-99$ & $0-47$ & $5-78$ \\
\hline \multicolumn{6}{|c|}{ CVC (days) } \\
\hline Mean & 2.97 & 1.07 & 15 & 11.69 & 29.31 \\
\hline Median & 0 & 5 & 16 & 6 & 33 \\
\hline Min-max & $0-44$ & $0-60$ & $0-60$ & $0-40$ & $0-53$ \\
\hline \multicolumn{6}{|c|}{ Vancomicine (days) } \\
\hline Mean & 1.34 & 15.26 & 20.17 & 11.44 & 18.92 \\
\hline Median & 0 & 14 & 21 & 10 & 17 \\
\hline Min-max & $0-21$ & $0-57$ & $4-46$ & $0-35$ & $9-37$ \\
\hline \multicolumn{6}{|c|}{ Meropenem (days) } \\
\hline Mean & 0.55 & .65 & 3.67 & 5.13 & 8.92 \\
\hline Median & 0 & 0 & 0 & 0.5 & 4 \\
\hline Min-max & $0-14$ & $0-51$ & $0-22$ & $0-21$ & $0-37$ \\
\hline \multicolumn{6}{|c|}{ Cefepime (days) } \\
\hline Mean & 0.84 & 5.88 & 2.75 & 4.25 & 6.54 \\
\hline Median & 0 & 1 & 0 & 1,5 & 4 \\
\hline Min-max & $0-14$ & $0-24$ & $0-15$ & $0-15$ & $0-20$ \\
\hline
\end{tabular}

Table 1: Descriptive statistics of the variables in each group. 


\begin{tabular}{|c|c|c|c|c|c|}
\hline & $\begin{array}{l}\text { Fungal sepsis versus no } \\
\text { fungal sepsis }\end{array}$ & $\begin{array}{l}\text { Fungal sepsis } \\
\text { versus no sepsis }\end{array}$ & $\begin{array}{c}\text { Fungal sepsis versus sepsis and } \\
\text { negative blood culture }\end{array}$ & $\begin{array}{l}\text { Fungal sepsis } \\
\text { versus } \mathrm{G}+\text { sepsis }\end{array}$ & $\begin{array}{l}\text { Fungal sepsis versus } \\
\text { G - sepsis }\end{array}$ \\
\hline & $p^{*}$ & $\mathrm{p}^{* *}$ & $\mathrm{P}^{* *}$ & $p^{\star *}$ & $\mathrm{P}^{* *}$ \\
\hline BW (g) & $<0.0001$ & $<0.0001$ & 0.576 & 0.022 & 0.496 \\
\hline GA (weeks) & $<0.0001$ & $<0.0001$ & 0.271 & 0.036 & 0.02 \\
\hline Fasting (d) & $<0.0001$ & $<0.0001$ & 0.002 & 0.023 & 0.001 \\
\hline$P N(d)$ & $<0.0001$ & $<0.0001$ & 0.005 & 0.017 & 0.008 \\
\hline$M V(d)$ & $<0.0001$ & $<0.0001$ & 0.011 & 0.023 & 0.009 \\
\hline CVC (d) & $<0.0001$ & $<0.0001$ & 0.002 & 0.016 & 0.009 \\
\hline Vancomicine(d) & $<0.0001$ & $<0.0001$ & 0.197 & 0.835 & 0.039 \\
\hline Meropenem (d) & $<0.0001$ & $<0.0001$ & 0.039 & 0.025 & 0.168 \\
\hline Cefepime (d) & $<0.0001$ & $<0.0001$ & 0.718 & 0.141 & 0.527 \\
\hline
\end{tabular}

${ }^{*} p<0.05$ (Kruskall-Wallis)

${ }^{* *} \mathrm{p}$ for multiple comparisons $\leq 0.005$ ( Bonferroni's test)

Table 2: Comparison of the risk factors considering: the presence and the absence of fungal infection and the comparison between the groups.

\begin{tabular}{|c|c|c|c|c|c|}
\hline \multirow[t]{2}{*}{ Group } & & \multirow[t]{2}{*}{$\mathbf{p}$} & \multirow[t]{2}{*}{ OR } & \multicolumn{2}{|c|}{ IC $95 \%$} \\
\hline & & & & IL & SL \\
\hline \multirow[t]{3}{*}{ No infection } & $B W(g)$ & 0.023 & 1.003 & 1.000 & 1.006 \\
\hline & MV & 0.007 & 0.895 & 0.825 & 0.97 \\
\hline & CVC (d) & 0.019 & 0.922 & 0.861 & 0.987 \\
\hline \multirow[t]{3}{*}{ Sepsis and negative blood culture } & $\mathrm{BW}(\mathrm{g})$ & 0.489 & 1.001 & 0.998 & 1.004 \\
\hline & MV & 0.467 & 1.012 & 0.979 & 1.046 \\
\hline & CVC (d) & 0.005 & 0.93 & 0.884 & 0.979 \\
\hline \multirow[t]{3}{*}{ G+ sepsis } & $\mathrm{BW}(\mathrm{g})$ & 0.017 & 1.004 & 1.001 & 1.006 \\
\hline & MV & 0.492 & 1.012 & 0.977 & 1.049 \\
\hline & CVC (d) & 0.045 & 0.943 & 0.891 & 0.999 \\
\hline \multirow[t]{3}{*}{ G- sepsis } & $\mathrm{BW}(\mathrm{g})$ & 0.687 & 1.001 & 0.997 & 1.004 \\
\hline & MV & 0.763 & 0.992 & 0.944 & 1,043 \\
\hline & CVC (d) & 0.065 & 0.94 & 0.88 & 1.004 \\
\hline
\end{tabular}

Table 3: Logistic regression of Birth Weight, Mechanical Ventilation and Central Venous Catheter (quantitative variables).

with the significant results. A final model was adjusted with just the risk factors that showed influence on the occurrence of fungal sepsis. Two models were used: the first considered BW, GA, fasting, PN, and MV as quantitative variables; the second used the qualitative variables (categorized). After a preliminary logistic regression using the quantitative variables, only BW, MV, and CVC remained as significant risk factors. Parenteral nutrition could not be included in the statistical model because all patients with fungal sepsis were exposed for more than seven days of PN.

In Table 3, assessing the risk of fungal sepsis in the different groups, we observed: 1) In the group without infection, the covariates that showed effects on the risk of fungal sepsis were BW, MV and CVC. The gain on average 10 grams in weight decreased the risk of fungal sepsis in $3.0 \%$. The increase of one day VPM increased risk of fungal sepsis in $11.1 \%$ and the increase of one day CVC increased the risk of fungal sepsis in $8.1 \%$. 2) Sepsis with negative blood cultures: an increase in one day of CVC increased the risk of fungal sepsis in $7.2 \%$. 3) Grampositive sepsis: $\mathrm{BW}$ and CVC were covariates that influenced the risk of fungal sepsis. The increase of 10 grams in PN decreased the risk of fungal sepsis in $4.0 \%$; the increase in CVC a day increased the risk of fungal sepsis in 5.8\%. 4) Gram-negative sepsis: there were no effects of covariates on the risk of fungal sepsis (the two infections were influenced by the same covariates).

In Table 4, assessing the risk of fungal sepsis, considering the categorization of variables, we observe: 1) In the group without infection, PN, VPM and CVC had effects on the risk of fungal sepsis; $\mathrm{BW} \leq 1000 \mathrm{~g}$ and $\mathrm{MV} \geq 14$ days increased by 23 times and 36 times the risk of fungal, respectively; the increase in CVC in a day increased the risk of fungal sepsis in 9.1\%. 2) In sepsis with negative blood cultures, $\mathrm{GA} \geq 28$ weeks decreased the risk of fungal sepsis compared to sepsis with negative blood cultures 16 times. 3) In gram-positive sepsis, BW $\leq 1000 \mathrm{~g}$ increased by 15 times the risk of fungal sepsis. 4) In gramnegative sepsis, the increase in a day-time exposure to CVC increased the risk of fungal sepsis in $7.5 \%$.

\section{Discussion}

The prevalence of late-onset sepsis (LOS) was 58.8\% with identification of the etiological agent in 52 cases. Gram-positive sepsis was found in $12.8 \%$, gram-negative in $8.5 \%$, and fungal sepsis in $7 \%$ with a mortality rate of $69.2 \%$. During the period of the study, fungal sepsis was the second most important agent of LOS. Other authors described the emergence of Candida spp as an agent of LOS during the past few years, and its prevalence ranged between $9 \%$ and $16 \%$ [1-8]. The risk factors associated with fungemia were birth weight, gestational age, prolonged fasting, parenteral nutrition, central venous catheters, mechanical ventilation, and the use of broad-spectrum antibiotics (Table 1).

Comparing the group with fungal sepsis with all remaining samples (no fungal sepsis), we observed that the risk factors associated with fungal sepsis were BW, GA, prolonged fasting, $\mathrm{PN}, \mathrm{CVC}, \mathrm{MV}$, and broad-spectrum antibiotics (Table 2). When we compared fungal sepsis with the no-sepsis group, we found the same risk factors. However, comparison of the fungal sepsis group with the other groups showed that the risk factors involved in fungal sepsis were the same as those 


\begin{tabular}{|c|c|c|c|c|c|}
\hline \multirow[t]{2}{*}{ Group } & & \multirow[t]{2}{*}{$\mathbf{p}$} & \multirow[t]{2}{*}{ OR } & \multicolumn{2}{|c|}{ IC $95 \%$ (OR) } \\
\hline & & & & IL & SL \\
\hline \multirow[t]{4}{*}{ No infection } & CVC (d) & 0.006 & 0.915 & 0.859 & 0.974 \\
\hline & $\mathrm{BW} \leq 1000 \mathrm{~g}$ & 0.003 & 23.092 & 2.892 & 184.37 \\
\hline & $\mathrm{GA} \leq 28 \mathrm{w}$ & 0.075 & 0.185 & 0.029 & 1.185 \\
\hline & $M V \geq 14 \mathrm{~d}$ & 0.009 & 36.364 & 2.451 & 539.495 \\
\hline \multirow[t]{4}{*}{ Sepsis and negative blood culture } & CVC (d) & 0.082 & 0.957 & 0.91 & 1.006 \\
\hline & $\mathrm{BW} \leq 1000 \mathrm{~g}$ & 0.159 & 4.156 & 0.573 & 30.116 \\
\hline & $\mathrm{GA} \leq 28 \mathrm{w}$ & 0.029 & 16.4 & 0.032 & 0.834 \\
\hline & $M V \geq 14 \mathrm{~d}$ & 0.087 & 8.508 & 0.734 & 98.593 \\
\hline \multirow[t]{4}{*}{ G + sepsis } & CVC (d) & 0.103 & 0.956 & 0.905 & 1.009 \\
\hline & $\mathrm{BW} \leq 1000 \mathrm{~g}$ & 0.012 & 15.146 & 1.828 & 125.467 \\
\hline & $\mathrm{GA} \leq 28 \mathrm{w}$ & 0.153 & 0.253 & 0.039 & 1.663 \\
\hline & $M V \geq 14 d$ & 0.381 & 3.161 & 0.241 & 41.463 \\
\hline \multirow[t]{4}{*}{ G- sepsis } & CVC (d) & 0.024 & 0.93 & 0.873 & 0.99 \\
\hline & $\mathrm{BW} \leq 1000 \mathrm{~g}$ & 0.350 & 2.768 & 0.328 & 23.368 \\
\hline & $\mathrm{GA} \leq 28 \mathrm{w}$ & 0.991 & 1.012 & 0.14 & 7.322 \\
\hline & $M V \geq 14 d$ & 0.690 & 1.711 & 0.122 & 24.085 \\
\hline
\end{tabular}

Table 4: Logistic regression of central venous catheter, birth weight, gestational age and mechanical ventilation (cathegorized variables).

for gram-positive sepsis, and almost the same were found in gramnegative sepsis and sepsis without an identified agent. These findings suggest that the VLBW infant is particularly prone to develop LOS during hospitalization.

An inverse relationship between BW/GA and the incidence of LOS was found in our study. The importance of birth weight as a risk factor for fungal sepsis is very well described. Although survival of VLBW infants and extremely low birth weight infants is increasing, LOS is considered a serious complication [8]. In our study, the group with fungal sepsis had a mean birth weight of $869.2 \mathrm{~g}$ and the mean BW in the group without sepsis was $1221.9 \mathrm{~g}$. Gestational age was significantly lower in the group with fungal sepsis than in the group without sepsis (mean $\mathrm{GA}=28.1$ weeks versus $\mathrm{GA}=31.7$ weeks).

The logistic regression (Table 3 ) showed that the risk factors associated with fungal sepsis were BW, MV, and CVC. Increasing one day of MV led to an increase of $11.1 \%$ for developing fungal sepsis, and increasing one day of CVC augmented it by $8.1 \%$. In the group with sepsis and a negative blood culture, each day of CVC increased the risk of fungal sepsis by $7.2 \%$.

All of these findings lead us to conclude that the very low birth weight infant and/or the extremely premature infant is prone to late onset sepsis, particularly fungal sepsis. The closest observation shows that the more susceptible group to develop fungal sepsis was composed of newborns with $\mathrm{BW} \leq 1000 \mathrm{~g}$. Comparing the group with grampositive sepsis and fungal sepsis, BW and CVC were risk factors for fungemia. A $10 \mathrm{~g}$ increase in BW reduced the risk of fungemia by $4 \%$; a one-day increase of CVC augmented the risk of fungemia by $5.8 \%$. Gram-negative sepsis and fungal sepsis were shown to suffer from the same influences.

When comparing the group without infection with the group with fungal sepsis, after the categorization of the variables, we showed that $\mathrm{BW} \leq 1000 \mathrm{~g}$ and $\mathrm{MV} \geq 14$ days increased the risk of fungal sepsis by 23 times and 36 times, respectively. A one-day increase of CVC augmented the risk of fungemia in newborns with BW $\leq 1000$ by $9.3 \%$. Considering the group with sepsis and negative blood culture, GA $\geq 28$ weeks decreased the chance of fungal sepsis by 16 times. BW $\leq 1000 \mathrm{~g}$ increased the risk of fungemia by 15 times when compared with grampositive sepsis. Moreover, for gram-negative sepsis, each day of CVC increased the risk of fungal sepsis by $7.5 \%$. A comparison of the fungal group and the group without infection showed that each day of CVC increased the risk of fungal sepsis to $8.5 \%$. For BW $\leq 1000 \mathrm{~g}$, this risk increased to $9.3 \%$.

The triad composed of fasting, parenteral nutrition, and central venous catheter seems an important step for late onset sepsis, particularly fungal sepsis. Other authors also associated higher incidence of sepsis in infants submitted to slow advancement of enteral feeds versus rapid advancement of enteral feeds [18]. The optimal feeding strategy should be considered to avoid long exposure to parenteral nutrition and central venous catheters.

In VLBW infants exposed to MV for $\geq 14$ days, each day of mechanical ventilation increased the risk of fungal sepsis by $11.7 \%$ compared with the group without sepsis. The risk increased 36 times in newborns with $\mathrm{BW} \leq 1000 \mathrm{~g}$. These results show the importance of MV as a risk factor for fungal sepsis. Perhaps the colonization of the respiratory tract occurs earlier in association with frequent physiotherapeutic procedures. Intubation itself is also difficult and the mucociliar cleaning of the airways increases colonization $[19,20]$. Strategies for less invasive assisted ventilation in the VLBW should be very important to reducing exposure to MV and its complications, particularly fungal sepsis.

The previous use of broad-spectrum antibiotics is often associated with fungal sepsis. We confirmed this hypothesis, by considering the group with fungal sepsis and all of the samples versus the group without sepsis. Stoll et al. described that $44 \%$ of VLBW infants with fungemia previously used vancomycin [2]. In our study, all newborns with fungal sepsis were exposed for at least 7 days to vancomycin, but we found no association with fungal sepsis when considering newborns with late onset sepsis. The use of third-generation cephalosporins is usually associated with fungal sepsis [21]. During the period of the study, we needed to change our first choice of drug for the treatment of LOS without an etiological agent identified based on the G profile of sensibility identified in our patients. Therefore, identifying any association with fungal sepsis and previous use of this medication was not possible.

We conclude that the risk factors for fungal sepsis in VLBW infants resemble many aspects of prematurity and are closely related to the 
needed procedures used for survival of this group of patients. Even with improvements in neonatal assistance, strategies for improving perinatal services and preventing prematurity and extremely low birth weight infants should be considered.

\section{Summary}

Fungemia is a severe complication during the neonatal period among very low birth weight infants (VLBW). The aim of this study was to describe the incidence of fungal sepsis and to analyze the risk factors among this population. Data were collected prospectively during a 25-month period among VLBW infants admitted at the Neonatal Intensive Care Unit (NICU) with more than 72 hours of life. Patients were divided into five groups based on the first positive blood culture: without sepsis; sepsis with negative blood culture; grampositive bacterial sepsis; gram-negative bacterial sepsis; and fungal sepsis. For statistical analyses, the Pearson test, the Kruskal-Wallis test, and a logistic regression model were used. The study included 187 newborns: 110 (58.8\%) had late-onset sepsis and 13 (7\%) had fungal sepsis. The mortality rate was $69.2 \%$. The risk factors identified during a comparison between the fungemia group and the non-fungemia group (remaining samples) were birth weight (BW), gestational age (GA), central venous catheter (CVC), parenteral nutrition (PN), fasting, mechanical ventilation (MV), and exposure to vancomycin, cefepime, meropenem, and amikacin. Further statistical analyses showed that for each $10 \mathrm{~g}$ increase in BW, the risk of fungemia decreased 3\%; each day of CVC increased this risk by $8.1 \%$; and each day of MV increased this risk by $11.1 \%$. The analyses of the categorized variables showed that $\mathrm{BW} \leq 1000 \mathrm{~g}$ increased the risk of fungemia by 23 times; $\mathrm{MV} \geq 14$ days increased the risk by 36 times; and each day of CVC increased the risk of fungemia by $9.3 \%$.

\section{References}

1. Stoll BJ, Gordon T, Korones SB, Shankaran S, Tyson JE et al. (1996) Lateonset sepsis in very low birth weight neonates: a report from the National Institute of child health and human development neonatal research network. J Pediatr. 29: 63-71.

2. Stoll BJ, Hansen N, Fanaroff AA, Wright LL, Carlo WA, et al. (2002) Late-onset sepsis in very low birth weight neonates: the experience of the NICHD Neonatal Research Network. Pediatrics 110: 285-291.

3. Chapman RL (2003) Candida infections in the neonate. Curr Opin Pediatr 15 97-102.

4. Kossoff EH, Buescher ES, Karlowicz MG (1998) Candidemia in a neonatal intensive care unit: trends during fifteen years and clinical features of 111 cases. Pediatr Infect Dis J 17: 504-508.

5. Gaynes RP, Edwards JR, Jarvis WR, Culver DH, Tolson JS, et al. (1996)
Nosocomial infections among neonates in high-risk nurseries in the United States. National Nosocomial Infections Surveillance System. Pediatrics 98 357-361.

6. Fridkin SK, Kaufman D, Edwards JR, Shetty S, Horan T (2006) Changing incidence of Candida bloodstream infections among NICU patients in the United States: 1995-2004. Pediatrics 117: 1680-1687.

7. Clerihew L, Lamagni TL, Brocklehurst P, McGuire W (2006) Invasive funga infection in very low birthweight infants: national prospective surveillance study. Arch Dis Child Fetal Neonatal Ed 91: F188-192.

8. Stoll BJ, Hansen N (2003) Infections in VLBW infants: studies from the NICHD Neonatal Research Network. Semin Perinatol 27: 293-301.

9. Saiman L, Ludington E, Pfaller M, Rangel-Frausto S, Wiblin RT, et al. (2000) Risk factors for candidemia in Neonatal Intensive Care Unit patients. The National Epidemiology of Mycosis Survey study group. Pediatr Infect Dis J 19: 319-324.

10. Saiman L, Ludington E, Dawson JD, Patterson JE, Rangel-Frausto S, et al (2001) Risk factors for Candida species colonization of neonatal intensive care unit patients. Pediatr Infect Dis J 20: 1119-1124.

11. Kaufman DA, Gurka MJ, Hazen KC, Boyle R, Robinson M, et al. (2006) Patterns of fungal colonization in preterm infants weighing less than 1000 grams at birth. Pediatr Infect Dis J 25: 733-737.

12. El-Masry FA, Neal TJ, Subhedar NV (2002) Risk factors for invasive fungal infection in neonates. Acta Paediatr 91: 198-202.

13. Critchley IA, Douglas LJ (1985) Differential adhesion of pathogenic Candida species to epithelial and inert surfaces. FEMS Microbiol Lett 28: 199-203.

14. Bendel CM (2003) Colonization and epithelial adhesion in the pathogenesis of neonatal candidiasis. Semin Perinatol 27: 357-364.

15. Chien LY, Macnab Y, Aziz K, Andrews W, McMillan DD, et al. (2002) Variations in central venous catheter-related infection risks among Canadian neonata intensive care units. Pediatr Infect Dis J 21: 505-511.

16. Opilla M (2008) Epidemiology of bloodstream infection associated with parenteral nutrition. Am J Infect Control 36: S173.

17. Goldstein B, Giroir B, Randolph A; International Consensus Conference on Pediatric Sepsis (2005) International pediatric sepsis consensus conference: definitions for sepsis and organ dysfunction in pediatrics. Pediatr Crit Care Med 6: $2-8$.

18. Härtel C, Haase B, Browning-Carmo K, Gebauer C, Kattner E, et al. (2009) Does the enteral feeding advancement affect short-term outcomes in very low birth weight infants? J Pediatr Gastroenterol Nutr 48: 464-470.

19. Rowen JL, Rench MA, Kozinetz CA, Adams JM Jr, Baker CJ (1994) Endotracheal colonization with Candida enhances risk of systemic candidiasis in very low birth weight neonates. J Pediatr 124: 789-794.

20. Makhoul IR, Kassis I, Smolkin T, Tamir A, Sujov P (2001) Review of 49 neonates with acquired fungal sepsis: further characterization. Pediatrics 107: 61-66.

21. Benjamin DK Jr, DeLong ER, Steinbach WJ, Cotton CM, Walsh TJ, et al. (2003) Empirical therapy for neonatal candidemia in very low birth weight infants. Pediatrics 112: 543-547. 